IAA 2002-3139

\title{
CONSTRAINED AEROTHERMODYNAMIC DESIGN OF HYPERSONIC VEHICLES
}

\author{
Tom Gally* \\ Embry-Riddle Aeronautical University \\ Prescott, Arizona \\ Dick Campbell ${ }^{\dagger}$ \\ NASA Langley Research Center \\ Hampton, Virginia
}

\begin{abstract}
An investigation was conducted into possible methods of incorporating a hypersonic design capability with aerothermodynamic constraints into the CDISC aerodynamic design too. The work was divided into two distinct phases: develop relations between surface curvature and hypersonic pressure coefficient which are compatible with CDISC's direct-iterative design method; and explore and implement possible methods of constraining the heat transfer rate over all or portions of the design surface. The main problem in implementing this method has been the weak relationship between surface shape and pressure coefficient at the stagnation point and the need to design around the surface blunt leading edge where there is a slope singularity. The final results show that some success has been achieved, but further improvements are needed.
\end{abstract}

\section{Introduction}

The CDISC aerodynamic design method (Ref 1) has been an effective tool used in the design of aerodynamic surface and for a wide range of conditions from subsonic to supersonic flow (Ref. 1-3). It was felt that a valuable addition to this code would be the added ability to handle hypersonic flows with appropriate aerothermodynamic constraints. The specific class of hypersonic vehicles being considered is those blunt nosed re-entry bodies such as the Space Shuttle, X-37 and X-33. These geometries result in the classical hypersonic problem of mixed subsonic and supersonic flow with body conformal shock waves on the windward surface.

\footnotetext{
* Associate Professor, Department of Aerospace Engineering, Senior Member AIAA.

$\dagger$ Senior Research Engineer, Senior Member AIAA. Copyright $(C) 2002$ by the American Institute of Aeronautics and Astronautics, Inc. All rights reserved
}

The CDISC design tool developed at NASA Langley research center stands for Constrained Direct Iterative Surface Curvature and refers to the design methodology used by this method. The core of this method is a set of linear aerodynamic relations between the flow properties of velocity and pressure to the local surface curvature. These relations allow for rapid surface modifications to drive a give surface towards a specified distribution of pressures/velocities. On top of this basic capability are added constraints based upon geometry, desirable pressure features, total integrated forces, and others. These constraints have been developed by specialists in aerodynamic design and are based upon good design practices. CDISC has been effectively used to perform aerodynamic design subsonic, transonic and supersonic flows for both simple 2-D cases and complex 3-D aircraft geometries. As importantly, CDISC is largely independent of the CFD solution method requiring only an interface to exchange surface flow properties and return surface geometries. To date, CDISC has been implemented with a number of CFD codes including CFD3D, TLNS3D, FLOMG, and OVERFLOW. The latter code is the one used in this investigation.

To implement the desired changes in CDISC, two distinct research phases were needed. First, the appropriate hypersonic surface curvature-pressure design rules suitable for use within the CDISC logic needed to be developed and tested. Second, the ability to implement aerothermodynamic design constraints, in this case aero-heating, needed to be developed and also tested on typical surface geometries.

\section{$\underline{\text { Surface Curvature }-\mathrm{C}_{\mathrm{p}} \text { Relation }}$}

The basic flow relations embedded in CDISC are based upon linearized aerodynamic theory. Thus, for supersonic flow, by small perturbation theory, the pressure coefficient, $\mathrm{C}_{\mathrm{p}}$, and local surface angle are given by: 


$$
C_{p}=\frac{2 \theta}{\sqrt{M_{\infty}^{2}-1}}
$$

where the surface angle is defined in Fig. 1.

Or, with a small angle assumption and differentiating a relationship between pressure gradient and surface curvature is obtained.

$$
\frac{d C_{p}}{d x}=\frac{2}{\sqrt{M_{\infty}^{2}-1}} \frac{d^{2} y}{d x^{2}}
$$

CDISC uses this relation to drive the local surface towards the desired pressure profile. A similar relation is desired for hypersonic flow.

Lees' modified Newtonian theory (Ref. 4, pg 53) provides the simplest, relatively accurate method for predicting surface pressures on the windward side of hypersonic bodies. This theory relates surface pressure coefficient to the stagnation point value, $\mathrm{C}_{\mathrm{po}}$, and square of the sine of the surface slope as given below with the angles defined in Fig. 2.

$$
C_{p}=C_{p o} \sin ^{2}(\theta)
$$

Unfortunately, this relation is not linear and since the interest is in blunt hypersonic shapes, small angles cannot be assumed. However, after differentiation the following relation is obtained between $\mathrm{C}_{\mathrm{p}}$ gradients and surface curvature is obtained.

$$
\frac{d C_{p}}{d x}=\frac{2 \cos ^{2}(\phi)}{\tan (\theta)} \frac{d^{2} y}{d x^{2}}
$$

Note that the factor multiplying $\mathrm{d}^{2} \mathrm{y} / \mathrm{dx}^{2}$ will be zero at two locations: at the stagnation point where $\theta$ is $90^{\circ}$, and at the nose (or any vertical location) where $\phi=90^{\circ}$. In implementing this relation into a desire rule, traps will have to be used to avoid numerical difficulties at these "singularities".

Since Newtonian theory is not accurate on the leeward surface, a trigger was used to switch the design algorithm to that for supersonic flow in these regions at the location where the two design rules agree on the magnitude of the factor relating pressure gradient to surface curvature.

This hypersonic design rule was validated in 2-D using a standard hypersonic CFD test geometry for an elliptically shaped body. As mentioned, the CFD code OVERFLOW (Ref. 5) was used for the analysis in its 2$\mathrm{D}$, inviscid flow mode. Although this solution will not capture the chemical processes and non-equilibrium thermodynamic state of many hypersonic flow scenarios, it should provide a stable test bed for validating the design rules. All solutions to be shown are for a flight Mach number of 10 and at an angle-ofattack of 30 degrees. Typical Mach number contours for this solution are in Fig. 3 indicating the ability of the code to capture the hypersonic bow shock.

A second geometry to that shown, of similar shape, but with a significantly blunter nose shape was also generated. The validation test case was then to start with the thick geometry shape and use the design rule to drive the geometry towards the thinner shape using it's known pressure distribution. A comparison of the initial and target pressure distributions and geometries is given in Figs. 4 and 5 .

The design process was initiated by first obtaining a partially converged solution on the initial thick geometry (1200 iteration cycles) and then beginning an iteration process between running CDISC followed by 300 additional CFD cycles. A total of 18 of these design iterations were performed. The bulk of the design modifications occurred within the first 10 iterations, but additional design cycles were run to insure the stability and convergence ability of the design method. The final design results are shown in Figs. 6 and 7.

As can be seen, the lower surface matched the thin target shape (slope and curvature), but with a slight offset in both the surface and pressure distribution curve. Since CDISC drives the solution towards matching pressure gradients rather than pressure magnitudes, this result is not surprising. The differences appear to arise due to a problem matching the surface pressures in the immediate vicinity of the stagnation singularity point. In this region, the Newtonian theory predicts a very weak relation between surface shape and pressure coefficient. To account for this, a number of "acceleration" factors were investigated to speed up the rate at which the geometry was modified in this region. These factors are not based upon solid physical foundations and are still being tested to see the limits of their applicability.

Note that the upper, leeward surface matched the target pressures very well except at the cabin junction. This is to be expected to some extent due to the discontinuity in both surface slope and pressure gradients at this point. What is disappointing is the much greater difference between the target and final upper surface shape. This is an indication that the supersonic algorithm is less effective at hypersonic speeds due to the pressure coefficient approaching zero on the 
leeward surfaces. A switch to using velocity or Mach number matching rather than pressure matching should be considered to improve this situation.

\section{Constrained Heat Transfer Design}

The next phase of the research was to develop appropriate heat transfer constraints on top of the basic hypersonic design rule. This would give the designer the ability to specify heating rate limits or distributions without having to be concerned with actual pressure distributions with which they may or may not have experience with. Ideally, however, the constraint procedure should relate heating rates to the pressure distribution to achieve a cascading style design: i.e. specify or limit heating rates, modify the existing pressure distribution to achieve those rates, modify the surface geometry to achieve the target pressures. The value of using the pressure distribution as an intermediary is the fact that many of the existing CDISC constraints are also based Cp. Thus, implementing a heating rate constraint in this method insures it will be compatible with other desirable constraints such as body forces.

After a review of past, approximate heat rate solutions (Ref. 6 and 7), Van Dreist's hypersonic similarity solutions for cylinder/cone stagnation point heating rates (Ref. 4, pgs 253 and 254) were used as the basis for this development:

$$
q_{w}=C \operatorname{Pr}^{-0.6}\left(\rho_{e} \mu_{e}\right)^{1 / 2} \sqrt{\frac{d u_{e}}{d x}}\left(h_{a w}-h_{w}\right)
$$

While this relationship is complex, the important functionality for our purposes is that due to the gradient in edge velocity, $d u_{e} / d x$ that can be related to the pressure gradients.

$$
q_{w} \propto \sqrt{\frac{d u_{e}}{d x}}
$$

Using Euler's momentum equation and some linearization about the stagnation point location, Van Dreist's relation can be reduced to.

$$
\frac{d u_{e}}{d x} \propto \frac{1}{\left(C_{p o}-C_{p}\right)} \frac{d C_{p}}{d x}
$$

Thus the desire relationship between heating rate and pressures is:

$$
q_{w} \propto \sqrt{\frac{1}{\left(C_{p o}-C_{p}\right)} \frac{d C_{p}}{d x}}
$$

Note that this equation depends upon both the magnitude of the pressure coefficient and it's spatial gradient. In practice, the magnitude of the pressure coefficient is a week function of local surface shape, depending more upon the full geometry and the resulting shock stand off distance (which is neglected in Newtonian theory). Thus, to satisfy the desired heating rate, it is necessary to attempt to modify the gradient of the pressure coefficient instead.

Altogether, these rules define a constrained design method. First obtain a CFD solution for heating rate and pressure coefficient for a given body shape. Next modify the heating rate solution based upon design constraints. Using the design rules outlined above, modify sequentially the pressure distribution and surface geometry to obtain the desired heating rate. Restart the CFD solution with the new geometry and begin to iterate with the design method. The sequence of performing this constrained design is shown in Fig. 8.

This design method was validated in 2-D using the same body geometry as before. This time a viscous CFD solution was obtained using a body Reynolds number of 1 million and a fixed surface wall temperature of $540^{\circ} \mathrm{R}$. The heating rate solution for these flow conditions show a strong heating spike near the stagnation point as show by the results in Figs. 9 and 10. Note that the factor Qw is not the exact heat flux, but a related non-dimensional factor proportional to the surface temperature gradient. The test design problem was to eliminate this spike based upon a max heating rate limit.

After 18 design iterations, the heating is effectively reduced below the applied limit as shown below as seen in Fig. 12. While significant reductions in local heating were obtained, the corresponding changes in pressure coefficient are much smaller (Fig. 11). The only significant effect was a slight movement of the stagnation point aft.

When checking the surface geometry, the changes are even less pronounced (Fig. 13). This result indicates that the improved heating rates were obtained with only very subtle changes in the local surface slope and curvature just behind the nose on the lower surface.

The above development and validation shows that a CDISC hypersonic design rule is feasible and that successful constraints on surface heating rate can be build upon this rule. There does appear to be a limited ability of the method to design to target surface pressure values near the stagnation point, but this limitation does not appear to effect the ability to design to heating rates in the same location.

In addition, there is also a tendency when attempting to reduce heat flux in one area, to increase the heat flux in 
another. In retrospect, this could have been expected due to the fixed downstream points of the geometry. To reduce heating in one location my reducing the local surface curvature require an increased curvature elsewhere and thus an increased heat flux. A better method of design is to allow the entire surface to move and avoid this problem.

\section{Conclusions and Suggestions}

The goal of developing and demonstrating a hypersonic constrained design capability for CDISC has been met. The final methodologies are based upon straight forward, approximate relations that seem to provide adequate control of surface modifications to achieve the desired design objective. This control is weakest near the stagnation point of the flow due to the poor correlation between surface pressure and surface curvature in that region - a problem that also occurs in subsonic and transonic flow.

The application of a surface heat transfer constraint was also met with mixed results. The attempt to related surface pressures to heat fluxes appears to be successful. However, the utility of this constraint in an actual design process may be less than desired since the heat load appears to be redistributed by this process rather than alleviated. Further investigation of this tool for an applied design problem and in 3-D needs to be undertaken to determine the best application of this constraint ability.

Posible future work will be the extension and validation of these methods to 3-D geometries. In addition, a fuller set of constraints will be investigated as well as an improved method for designing on the leeward surfaces. Finally, more complex tests or applications will be studied involving designing with multiple constraints including geometry and/or force limits. Finally, a long-term goal is to implement this design method in a multi-point sense employing both hypersonic and subsonic flight conditions simultaneously.

\section{$\underline{\text { References }}$}

1) Campbell, R.L., "Efficient Viscous Design of Realistic Aircraft Configurations," AIAA Paper 982539, June 1998.

2) Milholen, W.E., II, "An Efficient Inverse Aerodynamic Design Method for Subsonic Flows," AIAA Paper 2000-0780, January 2000.

3) Yu, N.J., Campbell, R.L., "Transonic Airfoil and wing Design Using Navier-Stokes Codes," AIAA Paper 92-2651, 1992.

4) Anderson, J. D., Hypersonic and High Temperature Gas Dynamics, McGraw-Hill, New York, 1989.

5) Buning, Pieter, et al., “ Overflow User's Manual, Version 1.8," NASA Ames Research Center, February 1998.

6) DeJarnette, F.R., Cheatwood, R.M., "A Review of Some Approximate Methods Used in Aerodynamic Heating Analyses," Journal of Thermophysics, Vol. 1, No. 1, January 1987.

7) Harloff, G.J., Petrie, S.L., "Preliminary Aerothermodynamic Design Method for Hypersonic Vehicles," AIAA Paper 87-2545, August 1987. 


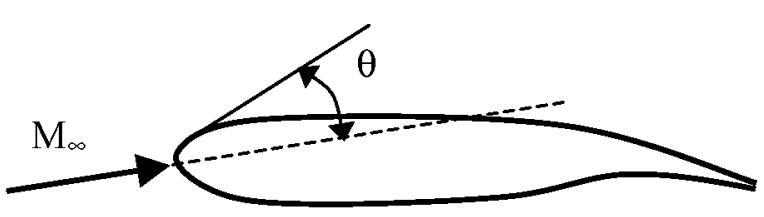

Figure 1: Geometry for Supersonic Small Perturbation Theory

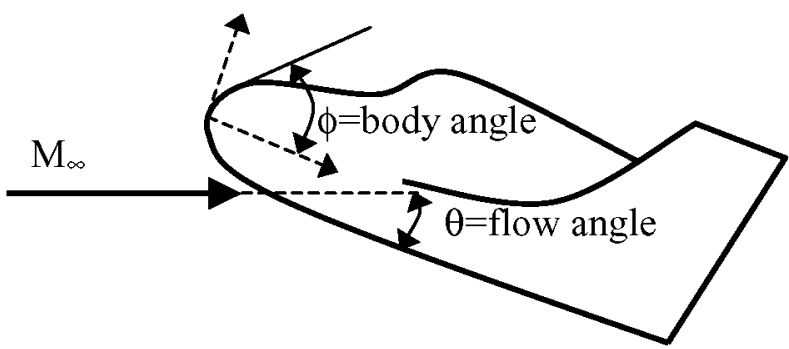

Figure 2: Geometry for Hypersonic Newtonian Flow Theory

Figure 3: Mach Contours for Typical Flow Case: $\mathrm{M}_{\infty}=10, \alpha=30^{\circ}$, inviscid.

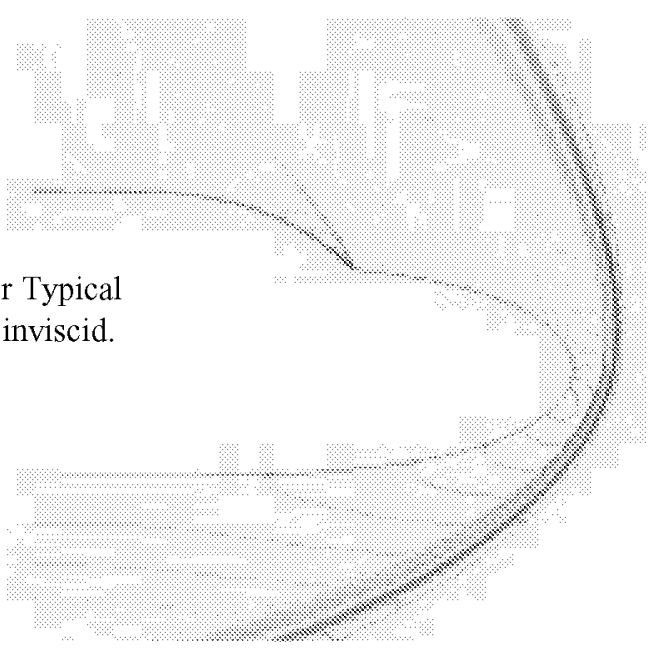

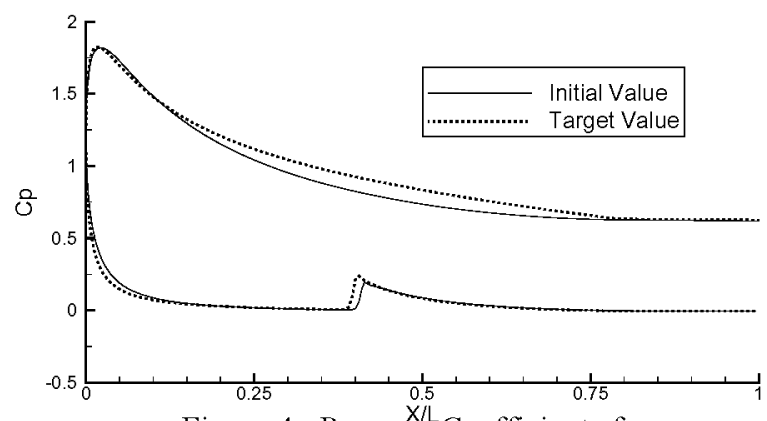

Figure 4: Pressure Coefficients for Initial and Target Cases

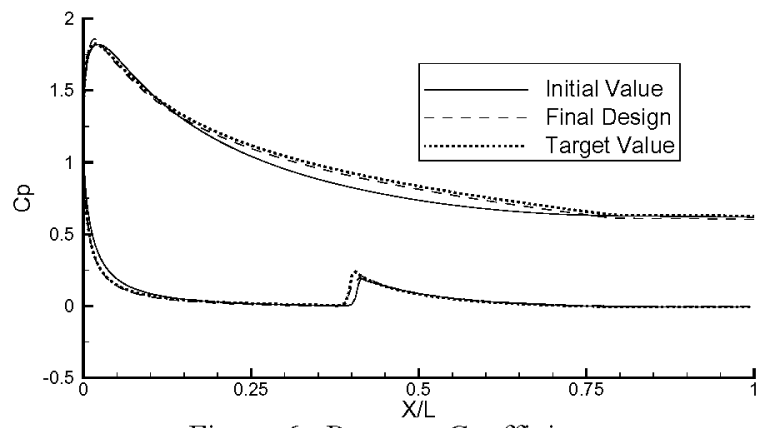

Figure 6: Pressure Coefficients Comparison for Final Design

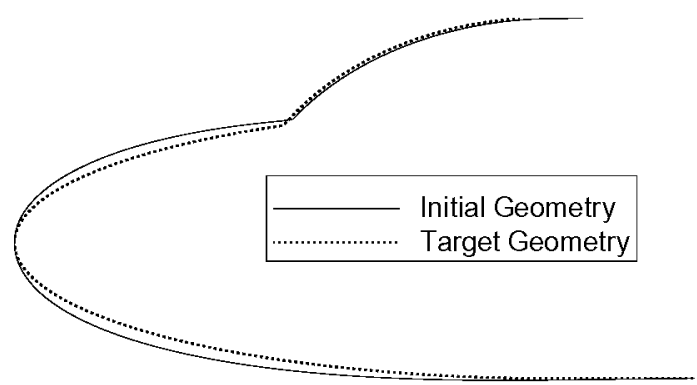

Figure 5: Surface Geometries for Initial and Target Cases

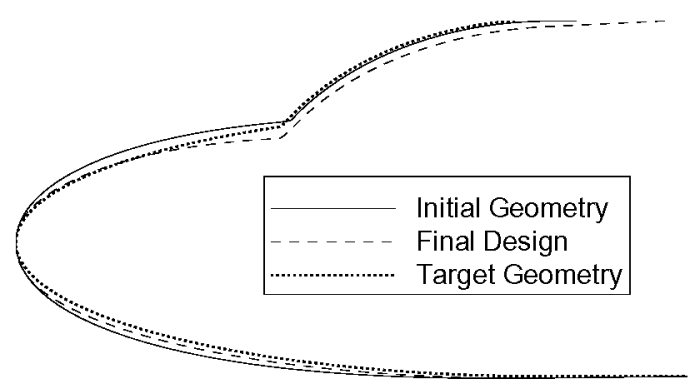

Figure 7: Surface Geometries Comparison for Final Design 


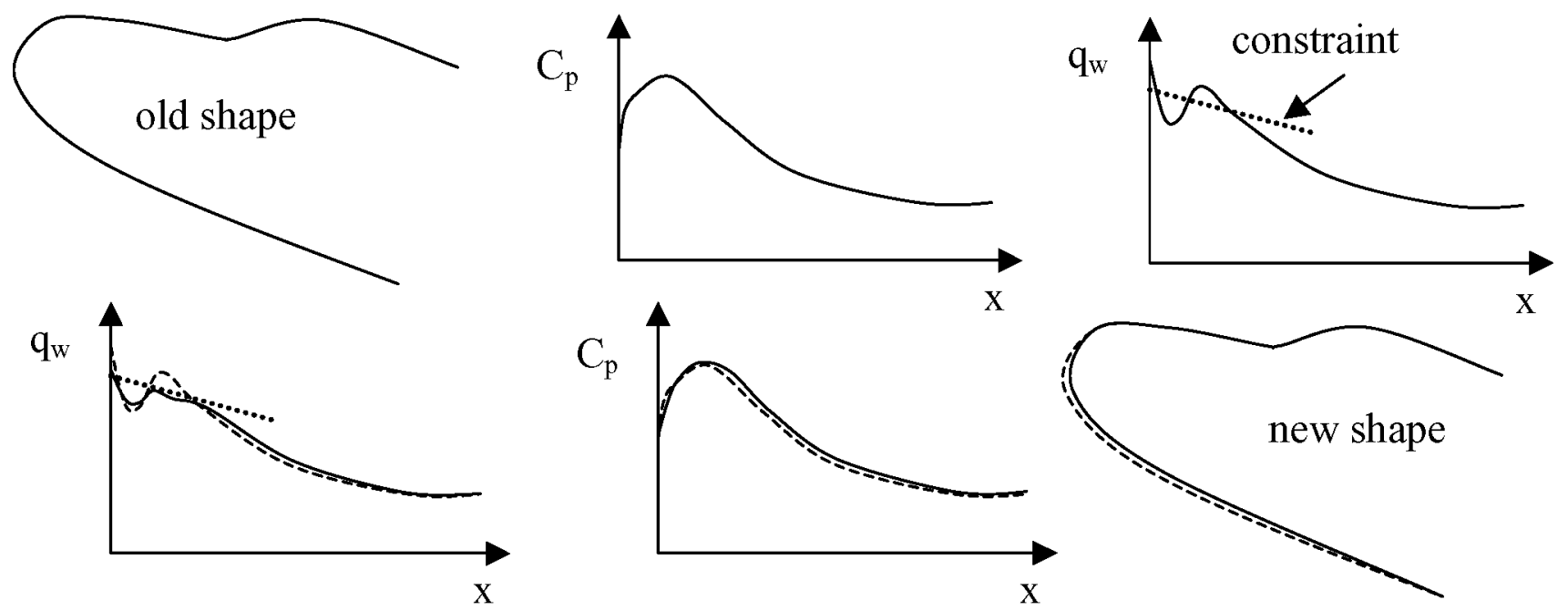

Figure 8: Overall Constrained Design

Sequence

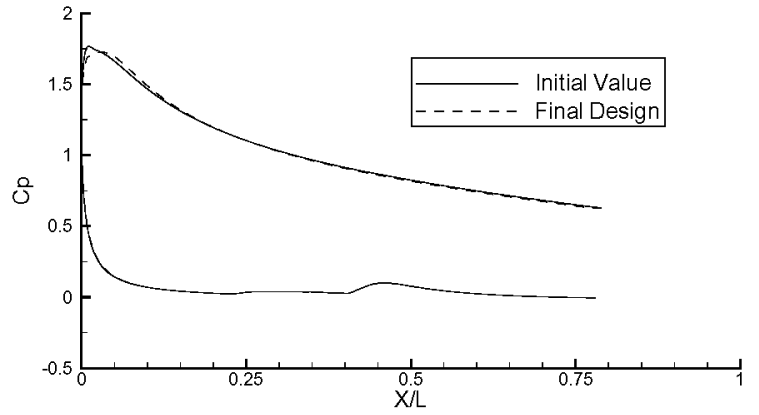

Figure 9: Initial Pressure Coefficients for Constrained Heat Transfer Test Case

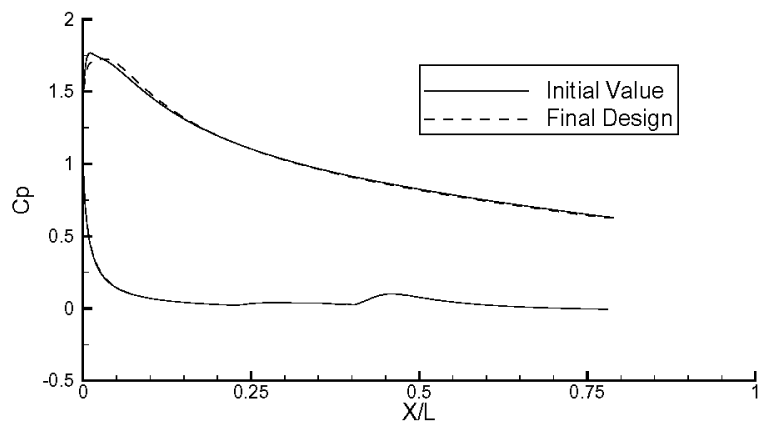

Figure 11: Pressure Coefficient Comparison for Final Design

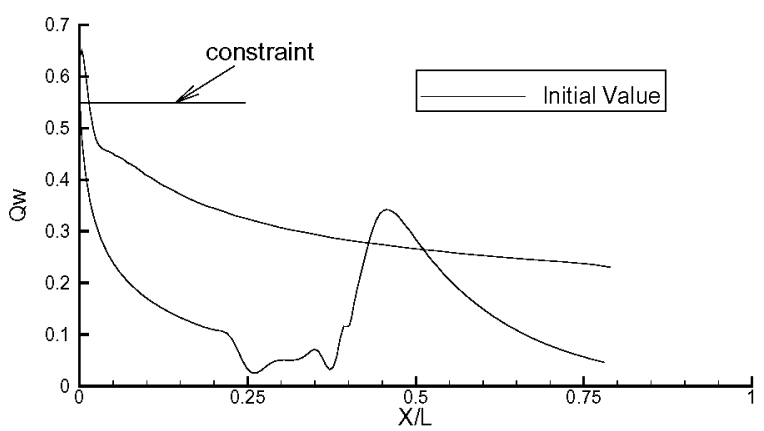

Figure 10: Initial Heat Fluxes for Constrained Heat Transfer Test Case

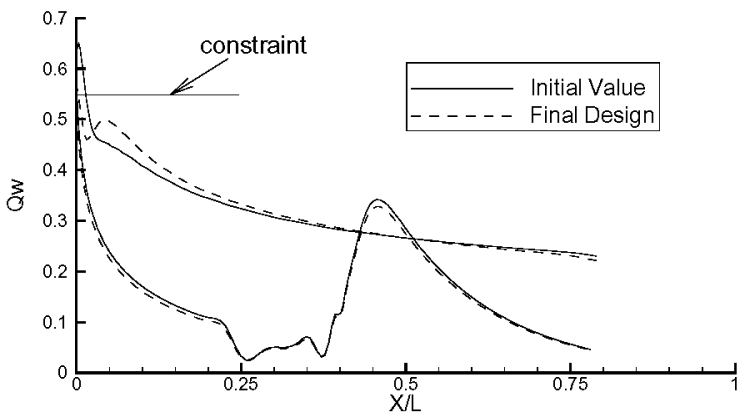

Figure 12: Heat Flux Comparison for Final Design 


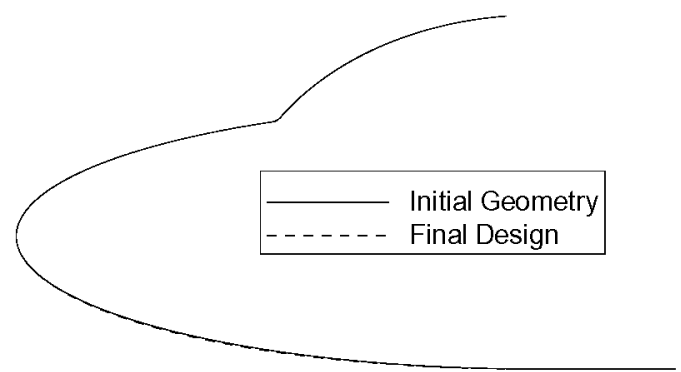

Figure 13: Surface Geometry Comparison for Final Design 